\title{
Juniperus turbinata Guss. (Cupressaceae), novedad para la flora de Extremadura (España)
}

José Alfredo Vicente Orellana \& Antonio Galán de Mera

Laboratorio de Botánica, Universidad CEU San Pablo, apartado 67, 28660 Boadilla del Monte, Madrid, España

\author{
Correspondencia \\ J.A. Vicente Orellana \\ e-mail: avicore@ceu.es \\ Recibido: 10 diciembre 2018 \\ Aceptado: 13 mayo 2019 \\ Publicado on-line: 18 septiembre 2019 \\ Editado por: A.V. Pérez Latorre
}

\begin{abstract}
Juniperus turbinata Guss. (Cupressaceae), a new record for the flora of in Extremadura (Spain)
\end{abstract}

Palabras clave: Extremadura, Juniperus turbinata, Península Ibérica, sabinares.

Key words: Extremadura, Juniperus turbinata, Iberian Peninsula, juniper schrublands.
Juniperus turbinata Guss. (sabina de costa) está ampliamente distribuida por la cuenca mediterránea, en general ligada a ambientes costeros, con suelos arenosos, entre los pisos infra- y mesomediterráneo -sobre todo en el termomediterráneo-, con ombroclima de semiárido a subhúmedo. No obstante, se tiene conocimiento de su presencia en algunas zonas de interior, como en la cordillera del Gran Atlas marroquí (Charco, 2001; Asensi et al., 2007; Adams, 2014), interior de Cerdeña (Bacchetta, 2006) y Sicilia (Gianguzzi et al., 2012). En el sur de la Península Ibérica también encontramos esta situación, tanto en Portugal -en laderas con fuerte pendiente cercanas al río Guadiana, sobre suelos ácidos (esquistos, grauvacas y cuarcitas)- (Capelo et al., 1994) como en España, en la Sierra de Grazalema -en calizas cámbricas- (Arista \& Ortiz, 1995), sierras interiores de Málaga -tanto sobre sustratos básicos (Díez Garretas et al., 1996) como en paleoislas y paleoacantilados silíceos (Pérez Latorre et al., 2006; Hidalgo \& Pérez Latorre, 2013), y sur de Sevilla -en margas yesíferas-- (Albarreal Núñez \& Romero Zarco, 2004). Su presencia en las áreas alejadas del mar se explicaría por la compensación entre el ombroclima semiárido y las bajas temperaturas, evitándose así la pérdida de la humedad que tendría cerca del mar (Mazur et al., 2016). A estas citas, añadimos esta nueva de Extremadura, que junto con las de Marruecos son las más interiores (Fig. 1):

ESPAÑA, Cáceres: Castañar de lbor, suelo pedregoso ácido-neutro, 30S288093-4398631, $650 \mathrm{~m}$, orientación SO, inclinación del suelo $40^{\circ}$,
25-II-2018, J.A. Vicente Orellana (USP3410).

El análisis de sus caracteres morfológicos y su fenología --hojas escuamiformes agudas, ápice de las ramas caudado, gálbulos ovoideos de más de $1 \mathrm{~cm}$, y floración otoñal--, confirma que se trata de J. turbinata (Franco, 1986), cuya presencia en esta localidad es muy probable que tenga que ver con su dispersión mediante aves migratorias (zorzales), y posiblemente en un futuro próximo su zoocoria esté también ligada a mamíferos

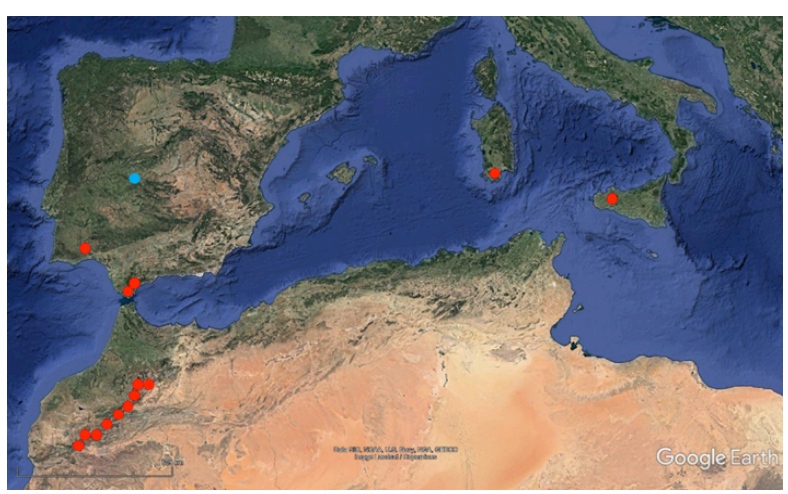

Figura 1. Mapa de distribución de las localidades interiores de Juniperus turbinata. Las localidades con punto rojo están basadas en los trabajos de Capelo et al., (1994), Arista \& Ortiz (1995), Bacchetta (2006), Gianguzzi et al., (2012), Hidalgo \& Pérez Latorre (2013), Mazur et al., (2018); El punto azul señala la nueva localidad (Fuente cartográfica: Google Earth).

Figure 1. Distribution map of inland localities of Juniperus turbinata. Red dots are localities from the bibliography; Blue dot is the new locality. (Cartographic base: Google Earth). 
(zorros) y aves (mirlos y zorzales), como sucede habitualmente en otras regiones (Adams, 2014). Se trata de una pequeña población con 1 ejemplar adulto (donde hemos podido levantar un inventario fitosociológico), y otros individuos más dispersos y de difícil acceso. Esta pequeña población se encuentran exactamente en el límite de la zona de protección ZEPA-ZEC "Sierra de las Villuercas y Valle de Guadarranque", por lo que se recomendaría estudiar la manera de incluirla, por su singularidad en la región.

La localidad se encuentra en el piso mesomediterráneo, con ombroclima subhúmedo, en una umbría, en medio de un madroñal espeso de la asociación Phillyreo angustifoliae-Arbutetum unedonis Rivas Goday \& F. Galiano in Rivas Goday, Borja, Esteve, Galiano, Rigual \& Rivas-Martínez 1960 (Fig. 2) sobre suelo pobre, pedregoso, que corresponde a tierras de raña con bloques, cantos y arcillas del Plioceno superior (IGME, 1986). Un inventario fitosociológico levantado en $25 \mathrm{~m}^{2}$ revela la siguiente composición. Características y diferenciales de asociación: Phillyrea angustifolia 2, Arbutus unedo 1, Viburnum tinus 2. Características de unidades superiores: Quercus suber 1, Quercus rotundifolia 1, Juniperus turbinata 1, Pistacia terebinthus 2, Erica arborea 1, Erica scoparia +,
Urginea maritima +. Compañeras: Cistus ladanifer 1, Rosmarinus officinalis 1, Lavandula luisieri 1, Erophaca baetica + , Melica uniflora 1, Thymus mastichina + , Helichrysum stoechas + , Rumex induratus + , Phagnalon saxatile + .

J. turbinata forma parte del complejo de Juniperus phoenicea $L$., que reúne a las especies mediterráneas $J$. phoenicea, J. turbinata y $J$. canariensis Guyot \& Mathou (Mazur et al., 2018). Se trata de un grupo relicto con un origen probable en el centro y oeste de Europa, y en especial en la Península lbérica, durante el Terciario. Según diferentes autores, al igual que el resto de la flora mediterránea, estas gimnospermas surgen durante el Oligoceno y tienen una diversificación posterior en el Pleistoceno (Lebreton \& Pérez de Paz, 2001; Mao et al., 2010; Fernández Palacios et al., 2011), por lo que sus enclaves con suelos pobres y condiciones xéricas (Charco, 2001; López González, 2001) pueden considerarse como refugios de la flora terciaria (Médail \& Diadema, 2009; Ivanov et al., 2011). En algunos lugares se ha podido observar el contacto entre J. phoenicea y J. turbinata, como sucede en el sur de la Península Ibérica (Arista \& Ortiz, 1995), si bien ambas permanecen aisladas genéticamente, ya que sus periodos de floración son muy diferentes, siendo

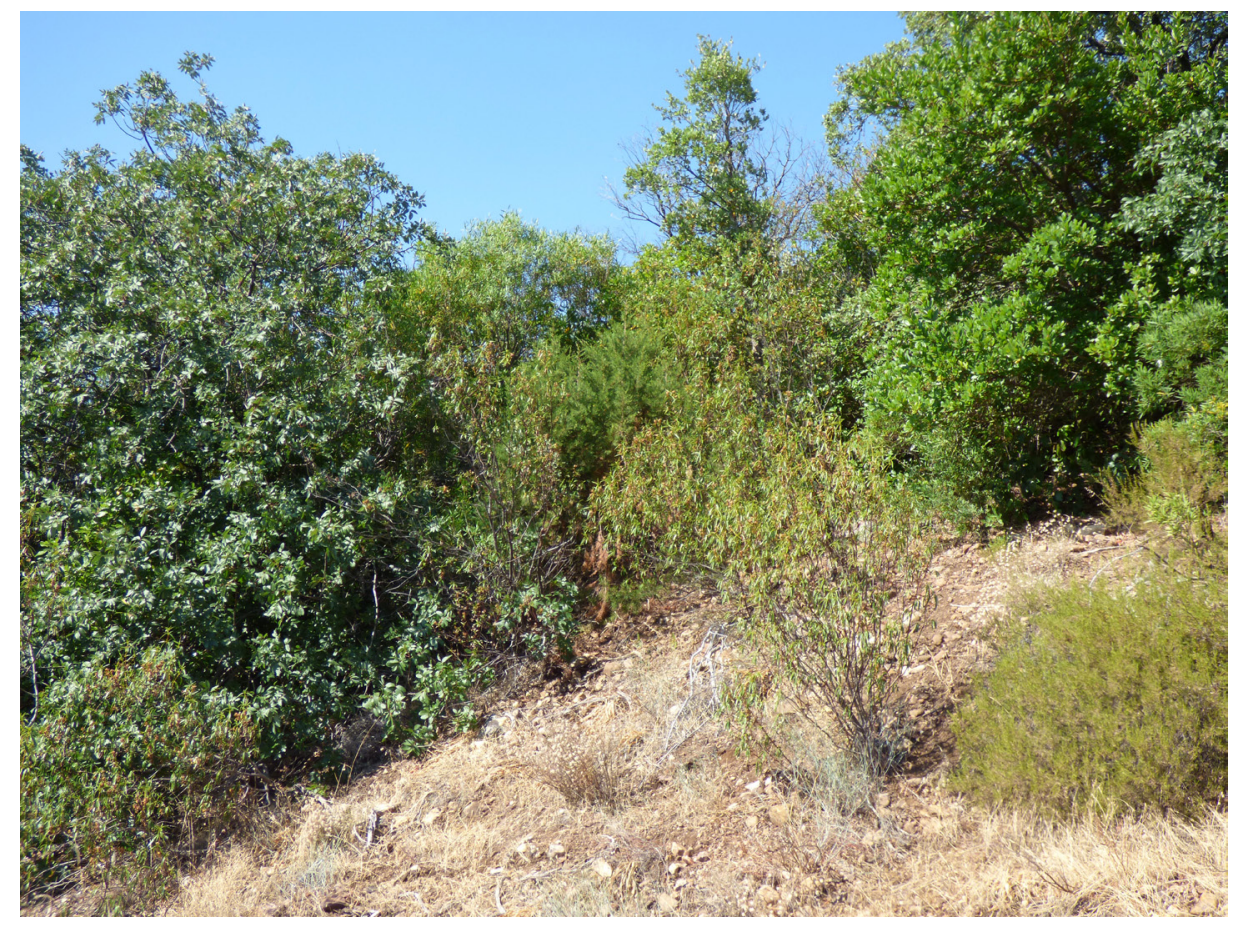

Figura 2. Imagen de Juniperus turbinata en el interior del madroñal de Phillyreo-Arbutetum unidonis en la nueva localidad de Extremadura.

Figure 2. Image of Juniperus turbinata inside the shrub of Phillyreo-Arbutetum unidonis at the new locality in Extremadura. 
primavera y otoño respectivamente (Boratyński et al., 2009).

\section{Bibliografía}

Adams, R.P. (2014). Junipers of the world: The genus Juniperus (4th ed.). Bloomington IN: Trafford Publications.

Albarreal Núñez, J., \& Romero Zarco, C. (2004). Novedades corológicas para Andalucía Occidental (Sevilla-Cádiz). Acta Botanica Malacitana, 29, 300304.

Arista, M., \& Ortiz, P.L. (1995). Juniperus phoenicea subsp. turbinata en La Sierra de Grazalema. Acta Botanica Malacitana, 20, 303-304.

Asensi, A., Díez-Garretas, B., \& Quézel, P. (2007). Plant communities of Juniperus turbinata Guss. subsp. turbinata in the Mediterranean Region. A biogeographical, bioclimatical and syntaxonomical survey. Phytocoenologia, 37, 599-623. DOI: 10.1127/0340-269X/2007/0037-0599

Bacchetta, G. (2006). Flora vascolare del Sulcis (Sardegna Sud-Occidentale, Italia). Guineana, 12, 1-369.

Boratyński, A., Lewandowski, A., Boratyńska, K., Montserrat, J.M., \& Romo, A. (2009). High level of genetic differentiation of Juniperus phoenicea (Cupressaceae) in the Mediterranean region: Geographic implications. Plant Systematic and Evolution, 277, 163-172. DOI: 10.1007/s00606-0080122-z

Capelo, J.H., Lousã, M.F., \& Costa, J.C. (1994). Phlomido purpureae-Juniperetum turbinatae, ass. nova: uma nova comunidade de Juniperus turbinata Guss. ssp. turbinata em xisto na bacia hidrográfica do rio Guadiana. Anais do Instituto Superior de Agronomia, 44, 515-530.

Charco, J. (2001). Guía de los árboles y arbustos del Norte de África. Madrid, MA: Agencia Española de Cooperación Internacional, Ministerio de Asuntos Exteriores.

Díez-Garretas, B., Asensi, A., \& Martín Osorio, V.E. (1996). Comportamiento fitosociológico de Juniperus phoenicia L. s.l. en el sur de la Península Ibérica. Lazaroa, 16, 159-167.

Fernández Palacios, J.M., de Nascimento, L., Otto, R., Delgado, J.D., García del Rey, E., Arévalo, J.R., \& Whittaker, R.J. (2011). A reconstruction of PalaeoMacaronesia, with particular reference to the longterm biogeography of the Atlantic island laurel forests. Journal of Biogeography, 38(2), 226-246. DOI: 10.1111/j.1365-2699.2010.02427.x

Franco, J.A. (1986). Juniperus L. In S. Castroviejo, M. Laínz, G. López González, P. Montserrat, F. Muñoz Garmendia, J. Paiva, \& L. Villar (Eds.), Flora iberica
(Vol. 1, pp. 181-188). Madrid, MA: Real Jardín Botánico.

Gianguzzi, L., Ilardi, V., Cardella, O., Cusimano, D., Cuttonaro, P., \& Romano, S. (2012). Phytosociological characterization of the Juniperus phoenicea L. subsp. turbinata (Guss.) Nyman formations in the ItaloTyrrhenian Province (Mediterranean Region). Plant Sociology, 49, 3-28. DOI: 10.7338/pls2012492/01

Hidalgo, N., \& Pérez Latorre A.V. (2013). Vegetación y flora de la Sierra de Cártama (Valle del Guadalhorce, Málaga, España). Acta Botanica Malacitana, 38, 119149.

Instituto Geológico y Minero de España (1986): Mapa geológico de España E. 1:200.000 (Talavera de la Reina). Madrid, MA: IGME.

Ivanov, D., Utescher, T., Mosbrugger, V., Syabryaj, S., Djordjević-Milutinović, D., \& Molchanoff, S. (2011). Miocene vegetation and climate dynamics in Eastern and Central Paratethys (Southeastern Europe). Palaeogeography, Palaeoclimatology and Palaeoecology, 304, 262-275. DOI: 10.1016/j. palaeo.2010.07.006

Lebreton, P., \& Pérez de Paz, P.L. (2001). Définition du Genévrier de Phénicie (Juniperus aggr. phoenicea), reconsidéré à ses limites biogéographiques: Méditerranée orientale (Créte et Chypre) et Atlantique (lles Canaries). Bulletin Mensuele de la Societe Linneenne de Lyon, 70(4), 73-92.

López González, G. (2001). Los árboles y arbustos de la Península Ibérica e Islas Baleares. 2 vol. MadridBarcelona-México: Mundi-Prensa.

Mao, K., Hao, G., Liu, J., Adams, R.P., \& Milne, R.I. (2010). Diversification and biogeography of Juniperus (Cupressaceae): Variable diversification rates and multiple intercontinental dispersals. New Phytologist, 185, 332-342. DOI: 10.1111/j.1469-8137.2010.03351.x

Mazur, M., Minissale, P., Sciandrello, S., \& Boratyński, A. (2016). Morphological and ecological comparison of populations of Juniperus turbinata Guss. and $J$. phoenicea $L$. from the Mediterranean region. Plant Biosystems, 150, 313-322. DOI: 10.1080/11263504.2014.994579

Mazur, M., Zielińska, M., Boratyńska, K., Romo, A., Salva-Catarineu, M., Marcysiak, K., \& Boratyński, A. (2018). Taxonomic and geographic differentiation of Juniperus phoenicea agg. based on cone, seed, and needle characteristics. Systematics and Biodiversity, 16, 469-482. DOI: 10.1080/14772000.2018.1439120

Médail, F., \& Diadema, K. (2009). Glacial refugia influence plant diversity patterns in the Mediterranean Basin. Journal of Biogeography, 36, 1333-1345. DOI: 10.1111/j.1365-2699.2008.02051.x

Pérez Latorre, A.V., Gavira, O., \& Cabezudo, B. (2006). Notas sobre la vegetación de Andalucía VII. Acta Botanica Malacitana, 31, 178-180. 